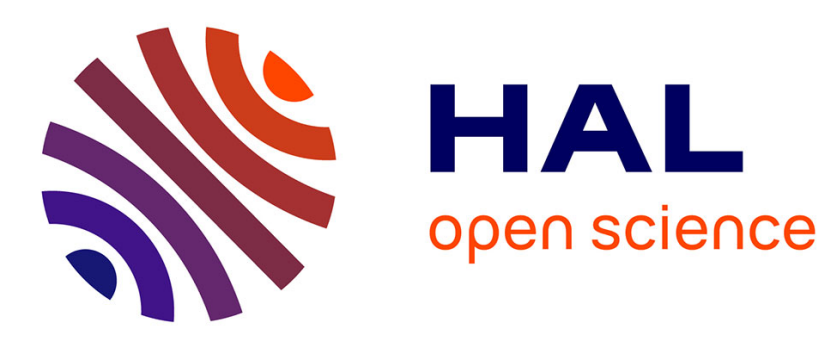

\title{
Fano Resonance Based Multiple Angle Retrodirective Metasuface
}

Mohammed Kalaagi, Divitha Seetharamdoo

\section{To cite this version:}

Mohammed Kalaagi, Divitha Seetharamdoo. Fano Resonance Based Multiple Angle Retrodirective Metasuface. EuCAP\&apos;20, 14th European Conference on Antennas and Propagation, Mar 2020, Copenhague, Denmark. 4p, 10.23919/EuCAP48036.2020.9135337 . hal-02926418v2

\section{HAL Id: hal-02926418 \\ https://hal.science/hal-02926418v2}

Submitted on 19 Apr 2021

HAL is a multi-disciplinary open access archive for the deposit and dissemination of scientific research documents, whether they are published or not. The documents may come from teaching and research institutions in France or abroad, or from public or private research centers.
L'archive ouverte pluridisciplinaire HAL, est destinée au dépôt et à la diffusion de documents scientifiques de niveau recherche, publiés ou non, émanant des établissements d'enseignement et de recherche français ou étrangers, des laboratoires publics ou privés. 


\title{
Fano Resonance Based Multiple Angle Retrodirective Metasuface
}

\author{
Mohammed Kalaagi ${ }^{1}$, Divitha Seetharamdoo ${ }^{1,2}$ \\ ${ }^{1}$ Institut de Recherche Technologique Railenium, F-59300, Famars, France \\ ${ }^{2}$ Univ Lille Nord de France, IFSTTAR, COSYS, LEOST, F-59650 Villeneuve d'Ascq, France \\ mohammad.kalaagi@railenium.eu, divitha.seetharamdoo@ifsttar.fr
}

\begin{abstract}
In this paper, a Fano resonance based multi-angle retrodirective metasurface is introduced. The aim is to investigate the potential of Fano-resonating structures, to suppress the losses and complexity of metasurface designs with high super-cell periodicities. A dolmen structure is given in this case which is well known for its Fano-resonating characteristics. It consists of two sub-radiant modes and one super-radiant mode. The design is given following the generalized phase law of reflection and surface impedance modulation for higher efficiency. The super-cell design has been given with a periodicity of $5.74 \lambda$, and frequency of 24 GHz. This is to achieve numerous retro-reflections at different incident angles based on Floquet harmonic analysis. When the sub-radiant mode is excited, retrodirectivity has been achieved at 17 angles of incidence. On the other hand, by exciting the superradiant mode, retrodirectivity has been achieved at three incident angles due to the coupling between the individual elements of the Fano-resonating structure. The monostatic RCS has been calculated to determine the performance of the retrodirective metasurface.
\end{abstract}

Index Terms-Fano resonance, retrodirectivity, multiple angles, generalized phase law, surface impedance modulation, Radar cross section enhacement.

\section{INTRODUCTION}

Metasurfaces [1] have gained lot of popularity in terms of wave control and manipulation for different applications [2][4]. Anomalous reflection has been proved to be achievable following the generalized phase law of reflection [5], that is, by introducing a phase gradient between scatterers, a metasurface can be designed to control an electromagnetic wave in a desired direction. Particularly for retro-reflection for a range of incident angles, this phenomenon has been in previous work [6], using the superposition of different designed super cells for different angles of incidence. It has also been reported by V.S. Asadchy et al [7], that with proper engineered surface impedance for a super-cell design periodicity which is greater than $\lambda$ according to Floquet harmonic analysis a multi-channel retro-reflective metasurface can be achieved [8].

However, with the increase of periodicity of up to 5.74 $\lambda$, the engineering of the surface impedance can become complex and the structure becomes very lossy. Sub-radiant resonances are well known to suppress radiation losses and overcome broad spectral responses. The interaction between the sub-radiant modes and the super radiant mode is known as Fano-resonance [9], [10], which results in a narrow spectral structure giving rise to a highly sensitive device at a selective frequency [11], [12]. These structures can be good candidates for applications such as nano sensing [13], imaging and highly sensitive devices [14].

In this paper we investigate the potential of Fano-resonating structures in order to suppress the losses in metasurfaces with high super-cell periodicities and to decrease the complexity of the optimization process to achieve multi-angle retrodirective metasurfaces using one super-cell design. A dolmen structure is introduced for its Fano-resonance properties [9]. The subradiant modes are tuned with a phase gradient following the generalized phase law of reflection with a fixed super-radiant mode. The coupling between the resonating structures of the dolmen unit cell, can give rise also to retro-reflection at some angles of incidence when the super-radiant mode is excited. A super-cell design of periodicity $5.74 \lambda$ is chosen to achieve retro-reflection for a numerous number of incident angles, since with respect to Floquet harmonic analysis [15], with an increase of periodicity greater than $\lambda$ more channels are present. In terms of application prospects, the multi-angle retrodirective metasurface can be a good candidate for highly sensitive radar systems [16] to increase the RCS of targets where backscattering is poor.

\section{Principle of Operation And Design Methdology}

In this work, we use dolmen fano-resonating structures, which are based on spectrum engineering for highly sensitive devices, and introducing a phase gradient by tuning the subradiant modes following the generalized phase law of reflection, particularly for retro-reflection, while fixing the superradiant mode. The unit cell structure is shown in Fig. 1.

The dimensions of the dolmen unit cell are given as follows: the two sub-radiant modes are of same dimensions in the $\mathrm{x}$ direction, $L_{1}=6.5 \mathrm{~mm}$ and $W_{1}=1 \mathrm{~mm}$. Whereas, for the superradiant mode in the y-direction, $L_{2}=2 \mathrm{~mm}$ and $W_{2}=1 \mathrm{~mm}$, with a gap $g=1 \mathrm{~mm}$ between the sub-radiant modes and the super-radiant modes.

The periodicity of the super-cell design, is determined following the integration of the generalized phase law of reflection [5] from 0 to $2 \pi$. The periodicity $L_{x}$ of the super cell design is given by:

$$
L_{x}=\frac{\lambda}{2 \sin \theta}
$$




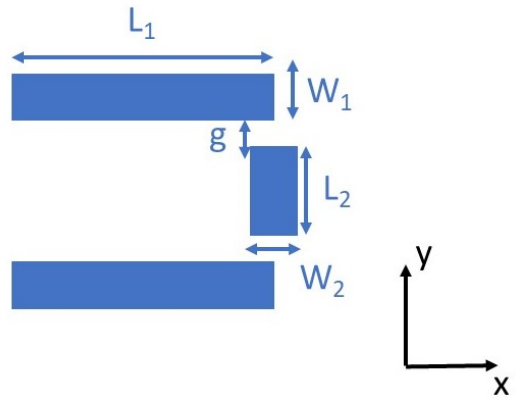

Fig. 1. Unit cell dolmen structure of 3 elements, two sub-radiant modes in the x-direction of dimensions $L_{1}$ and $W_{1}$, and one super-radiant mode in the y-direction of dimensions $L_{2}$ and $W_{2}$. With a gap g between the two sub-radiant modes and the super-radiant mode.

where the $\lambda$ is the wavelength and $\theta$ the angle of incidence. In our case, $\theta$ is chosen at $5^{\circ}$ for a frequency of $24 \mathrm{GHz}$, thus $L_{x}$ is equal to $5.74 \lambda$. According to Floquet Bloch analysis [15] for a metasurface design of periodicity larger than $\lambda$, a high number of open channels can exist where retrodirectivity can be achieved in addition to choosing the desired angle. For a periodicity of $5.74 \lambda$, the design becomes very lossy and complex in terms of optimization. The usage of a Fanoresonance structure can be a good candidate to suppress the losses for super-cells with high periodicities. The super-cell is divided into 8 unit cells, where the dimensions of the subradiant modes $L_{1}$ are both tuned similarly in the x-direction. The phase gradient $\phi=2 \pi / 8=45^{\circ}$. The dimensions of the sub-radiant modes are determined following the phase of the reflection coefficient which is shown in Fig. 2.

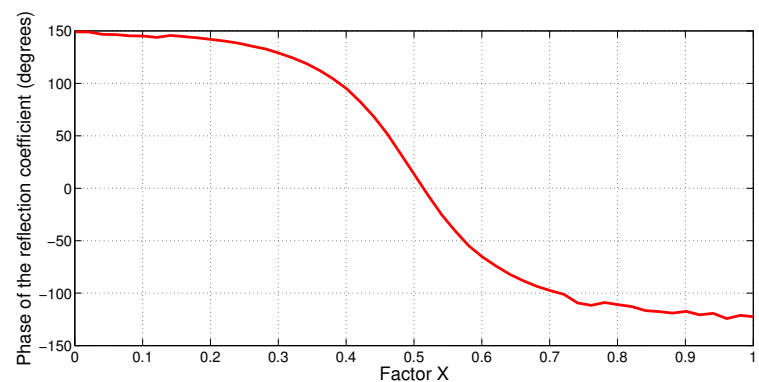

Fig. 2. Phase of the reflection coefficient with respect to that of Factor $X$ which is multiplied with the dimensions of the sub-radiant modes at $24 \mathrm{GHz}$.

The tuned dimensions are given as $L_{1(\text { initial })} \times$ Factor $\mathrm{X}$ with $L_{1 \text { (initial })}=6.5 \mathrm{~mm}$, which is determined in Fig. 2 with respect to a phase gradient $45^{\circ}$. The Factors $X$ and the dimensions of the tuned cells are given in Table 1.

In order to achieve high efficient retro-reflection at the open channels, the surface impedance should be properly engineered and optimized to be purely reactive, as given in the following equation:

$$
Z_{s}=j \frac{Z_{T M}}{\tan \left(\frac{\phi_{i}}{2}\right)}
$$

TABLE I

DIMENSIONS OF THE SUB-RADIANT MODES $L_{1}$ (MM) TUNED WITH A PHASE GRADIENT EQUAL TO $45^{\circ}$ FOLLOWING THE PHASE OF THE REFLECTION COEFFICIENT IN FIG. 2 , WHERE THE DIMENSIONS $L_{1 \text { (initial) }}$ $=6.5$ MM ARE MULTIPLED BY FACTOR X TO FORM A GRADIENT METASURFACE.

\begin{tabular}{lcr}
$\phi_{i}{ }^{\circ}$ & Factor $\mathrm{X}$ & $L_{1}(\mathrm{~mm})$ \\
\hline-122.4 & 1 & 6.5 \\
-77.4 & 0.6 & 3.9 \\
-32.4 & 0.55 & 3.575 \\
12.6 & 0.5 & 3.25 \\
57.6 & 0.44 & 2.86 \\
102.6 & 0.37 & 2.405 \\
147.6 & 0.1 & 0.65 \\
-167.4 & 1 & 6.5
\end{tabular}

where $Z_{s}$ is the surface impedance and $Z_{T M}$ is the impedance of air and $\phi_{i}$ is the phase of the reflection coefficient. The initail surface impedance of the dimensions given in Table 1 was calculated to be $\mathrm{Z}_{s}=15-j 326$ using Floquet boundary conditions for one super-cell design. The surface impedance has been optimized by tuning the initial dimensions to achieve a purely reactive surface impedance determined as $\mathrm{Z}_{s}=1-j 133$ where the resistance is almost tuned to zero. The optimized dimensions are given in Table 2.

TABLE II

OPTIMIZED AND INITIAL DIMENSIONS OF THE SUB-RADIANT MODES $L_{1}$ FOLLOWING THE SURFACE IMPEDANCE OPTMIZATION AND MODULATION TO A PURE IMAGINARY VALUE.

\begin{tabular}{cc} 
Initial Dimensions $L_{1}(\mathrm{~mm})$ & Optimized Dimensions $L_{1}(\mathrm{~mm})$ \\
\hline 6.5 & 6.5 \\
3.9 & 4.095 \\
3.575 & 3.705 \\
3.25 & 3.38 \\
2.86 & 3.055 \\
2.405 & 3.9 \\
0.65 & 1.3 \\
6.5 & 6.5
\end{tabular}

A metasurface of $4 \times 3$ super-cells of dimensions $25 \mathrm{~mm}$ $\times 215 \mathrm{~mm}$ is designed using the optimized dimensions given in Table 2. The dolmen structures are printed on top of a grounded substrate of permittivity $\varepsilon_{r}=2.2$ and thickness 1.6 $\mathrm{mm}$ operating at $24 \mathrm{GHz}$. The metasurface is shown in Fig 3.

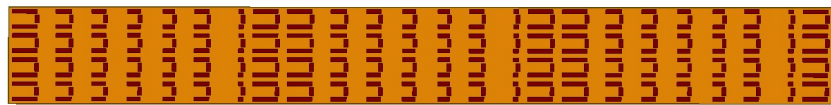

Fig. 3. Metasurface design using the optimized dimensions of $4 \times 3$ supercells, of dimensions $25 \mathrm{~mm} \times 215 \mathrm{~mm}$, with a ground plane and substrate of permittivity $\varepsilon_{r}=2.2$ and thickness $1.6 \mathrm{~mm}$.

The metasurface has been excited with a plane wave with the electric field in the X-direction of the sub-radiant modes, at a range of incident angles from $-70^{\circ}$ to $70^{\circ}$. The monostatic RCS has been calculated to determine the performance of the metasurface Fano-resonance based design. The monostatic RCS of the metasurface has been compared to that of a 
flat metallic plate with similar dimensions to highlight the efficiency of the metasurface for retrodirectivity. The result is shown in Fig.4.

The result shows high level of retrodirectivity for almost 17 different incident angles $\left(-60^{\circ},-52^{\circ},-44^{\circ},-38^{\circ}\right.$,$32^{\circ},-21^{\circ},-18^{\circ},-10^{\circ},-5^{\circ}$, normal incidence, $+5^{\circ},+18^{\circ}$, $+21^{\circ},+25^{\circ},+32^{\circ},+44^{\circ},+52^{\circ}$ ) which is due to the high periodicity of the super-cell design and high sensitivity of the designed Fano-resonant dolmen structure for high efficiency and accuracy.

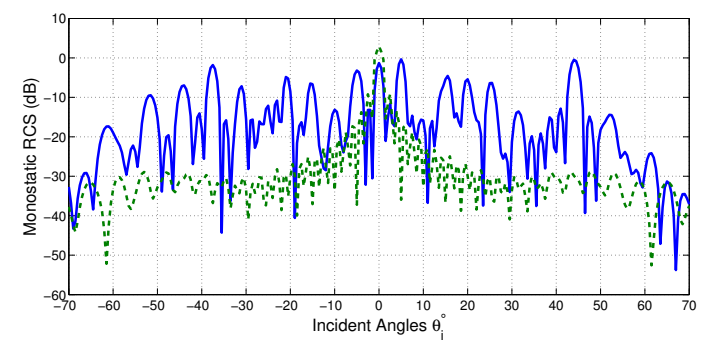

Fig. 4. Monostatic RCS $(\mathrm{dB})$ of the metasurface design in blue compared to a flat metallic plate of similar dimensions in dotted green, when the electric field of plane wave is in the direction of the sub-radiant modes (x-direction) at $24 \mathrm{GHz}$.

The metasurface has also been excited with a plane wave with the electric field in the direction of the fixed superradiant mode in the $y$-direction. The monostatic RCS has been calculated and compared to that of a metallic flat plate.

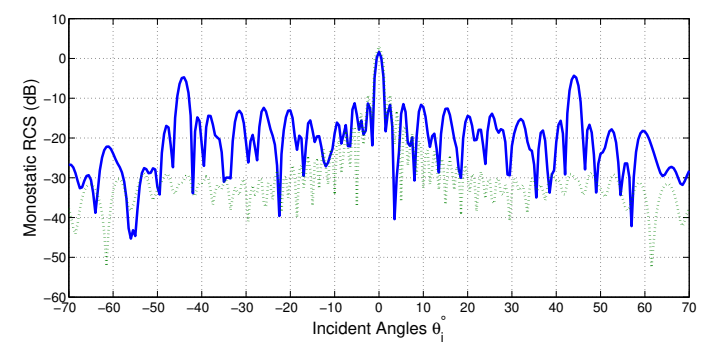

Fig. 5. Monostatic RCS (dB) of the metasurface design in blue compared to a flat metallic plate of similar dimensions in dotted green, when the electric field of plane wave is in the direction of the super-radiant mode (y-direction) at $24 \mathrm{GHz}$.

The result shows a high level of retro-reflection at three angles of incidence $-44^{\circ}$, normal incidence and $44^{\circ}$ compared to that of a flat plate of similar dimensions. This is due to the coupling present between the sub-radiant modes and the super-radiant mode, giving rise for retro-reflection for different angles with independent polarization for specific angles of incidence.

\section{CONCLUSION}

In this paper a retrodirective metasurface with high periodicity super-cell design, operating for multiple incident angles based on Fano-resonance has been introduced. The Fanoresonant metasurface design, has shown high sensitivity and efficiency for multiple angles at the desired frequency. The concept was to suppress the losses and complexity of the design at high periodic super-cell design. The metasurface has shown to perform efficient retro-reflection for 17 angles on incidence simultaneously. On the other hand, when exciting the super-radiant mode, due to the coupling between the resonant structures of the sub-radiant modes and super-radiant mode, the metasurface has shown to perform retrodirectivity at three angles simultaneously giving rise to independent polarization metasurface design. In terms of application prospects, the Fano-resonant metasurface design can be a good candidate for highly sensitive radar systems for increasing the RCS of targets with poor backscattering.

In future work, breaking the symmetry of the sub-radiant modes can be interesting to investigate, in order to achieve multiple Fano-resonances. A prototype will be realised and the measurement results will be presented at the conference.

\section{ACKNOWLEDGMENT}

The authors would like to acknowledge both technical and financial support from SNCF Réseau. One of the authors acknowledge partial funding of this research work by the regional project ORIO in the framework of the ELSAT 2020 program co-financed by the European Union with the European Regional development fund, the French state and Hauts de France Regional council.

\section{REFERENCES}

[1] H.-T. Chen, A. J. Taylor, and N. Yu, "A review of metasurfaces: physics and applications," Reports on Progress in Physics, vol. 79, no. 7, p. 076401, 2016.

[2] S. Sun, K.-Y. Yang, C.-M. Wang, T.-K. Juan, W. T. Chen, C. Y. Liao, Q. He, S. Xiao, W.-T. Kung, G.-Y. Guo, L. Zhou, and D. P. Tsai, "High-efficiency broadband anomalous reflection by gradient metasurfaces," Nano Letters, vol. 12, no. 12, pp. 6223-6229, 2012, pMID: 23189928. [Online]. Available: http://dx.doi.org/10.1021/nl3032668

[3] A. Pors, M. G. Nielsen, R. L. Eriksen, and S. I. Bozhevolnyi, "Broadband focusing flat mirrors based on plasmonic gradient metasurfaces," Nano letters, vol. 13, no. 2, pp. 829-834, 2013.

[4] A. Pors, O. Albrektsen, I. P. Radko, and S. I. Bozhevolnyi, "Gap plasmon-based metasurfaces for total control of reflected light," Scientific reports, vol. 3, no. 2155, pp. 1-6, 2013.

[5] N. Yu, P. Genevet, M. A. Kats, F. Aieta, J.-P. Tetienne, F. Capasso, and Z. Gaburro, "Light propagation with phase discontinuities: generalized laws of reflection and refraction," science, vol. 334, no. 6054, pp. 333$337,2011$.

[6] M. Kalaagi and D. Seetharamdoo, "Multiangle retrodirective cascaded metasurface," Journal of Applied Physics, vol. 126, no. 10, p. 104901, 2019.

[7] V. Asadchy, A. Díaz-Rubio, S. Tcvetkova, D.-H. Kwon, A. Elsakka, M. Albooyeh, and S. Tretyakov, "Flat engineered multichannel reflectors," Physical Review X, vol. 7, no. 3, p. 031046, 2017.

[8] C. Shen, A. Díaz-Rubio, J. Li, and S. A. Cummer, "A surface impedancebased three-channel acoustic metasurface retroreflector," Applied Physics Letters, vol. 112, no. 18, p. 183503, 2018.

[9] C. Yan, K.-Y. Yang, and O. J. Martin, "Fano-resonance-assisted metasurface for color routing," Light: Science \& Applications, vol. 6, no. 7, p. e17017, 2017.

[10] N. Verellen, Y. Sonnefraud, H. Sobhani, F. Hao, V. V. Moshchalkov, P. V. Dorpe, P. Nordlander, and S. A. Maier, "Fano resonances in individual coherent plasmonic nanocavities," Nano letters, vol. 9, no. 4, pp. 1663 1667, 2009.

[11] N. S. King, L. Liu, X. Yang, B. Cerjan, H. O. Everitt, P. Nordlander, and N. J. Halas, "Fano resonant aluminum nanoclusters for plasmonic colorimetric sensing," ACS nano, vol. 9, no. 11, pp. 10 628-10 636, 2015. 
[12] A. E. Miroshnichenko, S. Flach, and Y. S. Kivshar, "Fano resonances in nanoscale structures," Reviews of Modern Physics, vol. 82, no. 3, p. 2257, 2010.

[13] N. S. King, L. Liu, X. Yang, B. Cerjan, H. O. Everitt, P. Nordlander, and N. J. Halas, "Fano resonant aluminum nanoclusters for plasmonic colorimetric sensing," ACS nano, vol. 9, no. 11, pp. 10628-10 636, 2015.

[14] S. Bakhti, N. Destouches, and A. V. Tishchenko, "Coupled mode modeling to interpret hybrid modes and fano resonances in plasmonic systems," ACS Photonics, vol. 2, no. 2, pp. 246-255, 2015.

[15] A. Epstein and G. V. Eleftheriades, "Floquet-bloch analysis of refracting huygens metasurfaces," Physical Review B, vol. 90, no. 23, p. 235127 , 2014.

[16] Y.-C. Guo, X.-W. Shi, and L. Chen, "Retrodirective array technology," Progress In Electromagnetics Research B, vol. 5, pp. 153-167, 2008. 